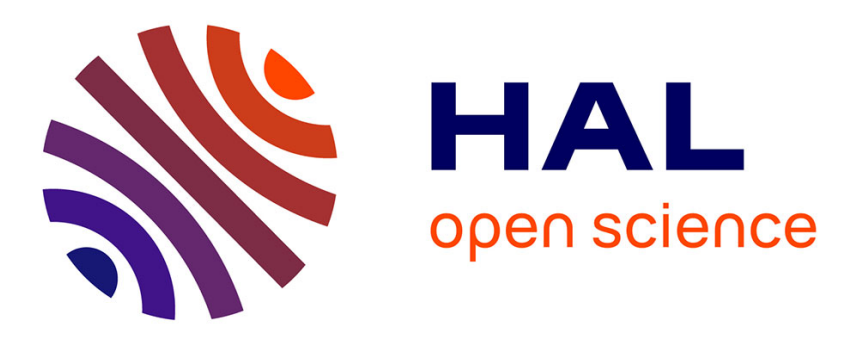

\title{
Angularly tunable bandpass filter: design, fabrication and characterization
}

Julien Lumeau, Fabien Lemarchand, Thomas Begou, Detlef Arhilger, Harro Hagedorn

\section{- To cite this version:}

Julien Lumeau, Fabien Lemarchand, Thomas Begou, Detlef Arhilger, Harro Hagedorn. Angularly tunable bandpass filter: design, fabrication and characterization. Optics Letters, 2019, 44 (7), pp.1829. 10.1364/OL.44.001829 . hal-02270080

\section{HAL Id: hal-02270080 \\ https://hal.science/hal-02270080}

Submitted on 23 Aug 2019

HAL is a multi-disciplinary open access archive for the deposit and dissemination of scientific research documents, whether they are published or not. The documents may come from teaching and research institutions in France or abroad, or from public or private research centers.
L'archive ouverte pluridisciplinaire HAL, est destinée au dépôt et à la diffusion de documents scientifiques de niveau recherche, publiés ou non, émanant des établissements d'enseignement et de recherche français ou étrangers, des laboratoires publics ou privés. 


\title{
Angularly tunable bandpass filter: design, fabrication and characterization
}

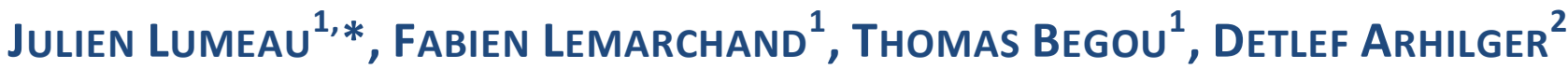 \\ AND HARRO HAGEDORN ${ }^{2}$ \\ ${ }^{1}$ Aix Marseille Univ, CNRS, Centrale Marseille, Institut Fresnel, Marseille, France \\ ${ }^{2}$ Bühler Leybold Optics, Alzenau, Germany \\ *Corresponding author: julien.lumeau@fresnel.fr
}

Received XX Month XXXX; revised XX Month, XXXX; accepted XX Month XXXX; posted XX Month XXXX (Doc. ID XXXXX); published XX Month XXXX

We present a thorough study of a bandpass filter for near-IR region, with bandwidth below $20 \mathrm{~nm}$, high transmission, broad rejection band [350-1100] $\mathrm{nm}$. This filter is angularly tunable between 0 and $50^{\circ}$ and shows a shift of the central wavelength from $970 \mathrm{~nm}$ down to $880 \mathrm{~nm}$ without major changes of its bandwidth and spectral shape for unpolarized light. We first provide a description of the design procedure and then carry out an experimental demonstration using plasma enhanced reactive magnetron sputtering. We finally present an accurate characterization of this class of filter using a custom optical system.

OCIS codes: (310.1620) Interference coatings; (310.1860) Deposition and fabrication; (310.4165) Multilayer design.

http://dx.doi.org/10.1364/OL.99.099999

Bandpass filters are among the most common complex spectral functions that can be achieved with thin-film optical interference filters. They are widely commercially available for various spectral regions from UV to IR regions [1-2]. The complexity of the filters has grown with the development of telecommunications filters for WDM (Wavelength Division Multiplexing) [3]. These filters are now used for a very broad range of applications including biophotonics [4], remote sensing, space and earth observation [5]. There have been numerous works that have been carried out in order to make these filters tunable. One can cite thermal tuning of bandpass filters [6], the combination of liquid crystal based systems with mirrors [7], acousto-optic tunable filters [8], or thinfilm Fabry-Perot filters combining active materials [9] or tuned by stress [10]. However, there is, as of today, no efficient method for the fabrication of tunable filters.

The most straightforward technique for tuning a filter consists in using the natural properties of the interference inside the layers and their large dependence on angle of incidence of the incoming light. Indeed, it is well-known that the spectral properties of any optical interference filter will shift towards shorter wavelength when angle of incidence is increased to larger angles [11]. However, spectrum is generally not maintained when the angle of incidence is changed and these distortions can be significantly different for $\mathrm{s}$ and $\mathrm{p}$ polarizations. This is easily explained by the fact that the optical admittance of each layer, $\eta$, which connects the electric and magnetic fields, is different for each polarization [11]. In order to circumvent this limitation, it is possible to design nonconventional structures and such filters are nowadays available [12]. However, there are little details about the way to achieve such high performance angularly tunable filters and characterization remains a challenge as the filter becomes more and more angularly selective when angle of incidence is increased.

In this paper, we present a thorough study of this class of filters. The specifications of the filter we studied are given in Table.1.

Table 1. Specifications of a tunable bandpass filter @ 970 nm

\begin{tabular}{|c|c|}
\hline Substrate & Fused silica \\
\hline High index material & $\mathrm{Nb}_{2} \mathrm{O}_{5}$ \\
\hline Low index material & $\mathrm{SiO}_{2}$ \\
\hline Central wavelength @0 & $970 \mathrm{~nm}$ \\
\hline${\text { Central wavelength @ } \mathbf{5 0}^{\circ}}^{\circ}$ & $880 \mathrm{~nm}$ \\
\hline Transmission in bandpass & $>90 \%$ \\
\hline FWHM & $<20 \mathrm{~nm}$ \\
\hline Polarization & Unpolarized light \\
\hline Edge steepness of bandpass & $\Delta \lambda<2 \mathrm{~nm}$ from $\mathrm{T}=10 \%$ to \\
& $80 \%$ \\
\hline Out of band rejection & $\mathrm{T}<1 \%$ from $350 \mathrm{~nm}$ to \\
& $1100 \mathrm{~nm}$ \\
\hline
\end{tabular}

First, we designed a structure that allows performing theses spectral performances. Let us now analyze these specifications from a design perspective. First, the broad blocking band [350$1100] \mathrm{nm}$ requires combining several blacking filters in order to cover the whole spectral range which will result in very thick coating. In order to limit the thickness of the coating, 2-side coating appears as recommended. The edge steepness between bandpass and blocking regions is $2 \mathrm{~nm}$; To achieve such sharp transmission change, a minimal thickness $\sim 20 \mu \mathrm{m}$ appears as mandatory and 
the most constraining conditions will probably be for 50 deg angle of incidence. Indeed, as the angular shift between $\mathrm{s}$ and $\mathrm{p}$ polarizations is the largest at $50^{\circ}$, having a constant transmission for an average polarization is very challenging. Thus, keeping the square shaped behavior from 0 to $50^{\circ}$ for both polarizations will require taking into account several angular targets, namely at 0,20 , 30,40 , and $50^{\circ}$ when performing the design. Finally, many parasitic peaks outside the bandpass are expected to appear. Some will be very thin $(<10 \mathrm{pm})$ and with high transmittance (about 50\%). When defining the target spectral profile, a very densified target grid will be mandatory to eliminate these peaks.

To perform the design, we decided to split the optical function into two parts associated with two-side coating. On the front side, we decided to produce the expected spectral profile associated with the [780-1100] nm spectral band and to associate with the rear side, blocking filter. This rear side does not present severe issues from a design point of view and will not be further discussed in this paper. Concerning the design of the front side coating, we defined a dense grid in [780-850] $\mathrm{nm}$ and [1000-1100] $\mathrm{nm}$ ranges. Only two materials were considered: $\mathrm{Nb}_{2} \mathrm{O}_{5} / \mathrm{SiO}_{2}$ as this is the couple of materials that provides the best refractive index contrast. The starting design was a non-optimized $20 \mu \mathrm{m}$ design ( $\sim 80$ layers) which enables many possibilities to insert needles and needle optimization technique was implemented using Optilayer software and densification of the target grid when needed in order to eliminate parasitic peaks [13].

The main parameters of the designed filter on front face are provided in Table 2 and the spectral profiles for an angle of incidence of 0, 20, 30, 40 and $50^{\circ}$ are shown in Figure 1 . On can see that high performance angularly tunable filter can be achieved with this design.

Table 2. Typical specifications of the designed front face

\begin{tabular}{|c|c|}
\hline Total thickness & $20.3 \mu \mathrm{m}$ \\
\hline Thickness $\mathbf{N b}_{\mathbf{2}} \mathbf{O}_{\mathbf{5}}$ & $9.2 \mu \mathrm{m}$ \\
\hline Thickness $\mathbf{S i}_{2}$ & $11.1 \mu \mathrm{m}$ \\
\hline Minimal thickness & $51 \mathrm{~nm}$ \\
\hline Number of layers & 141 \\
\hline
\end{tabular}

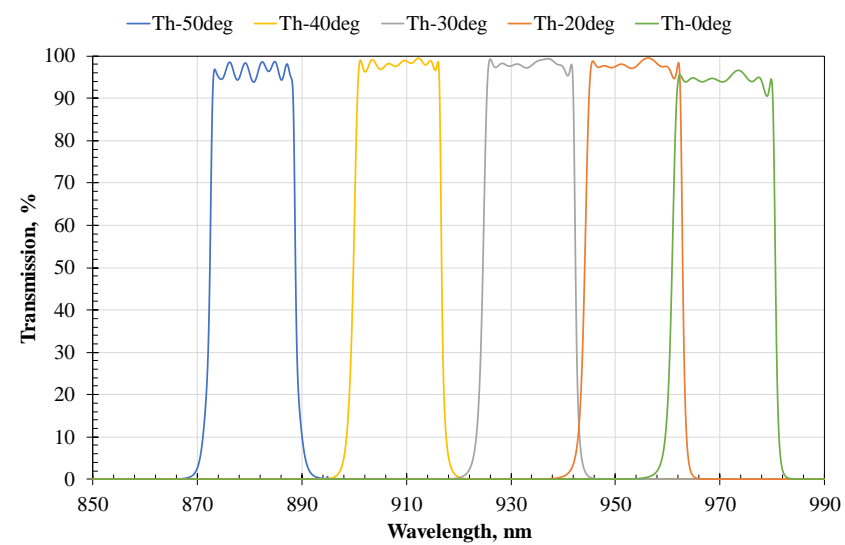

Fig. 1. Spectral dependence of the transmission of the designed bandpass for different angles of incidence.

In Figure 2, we show a zoom on the bandpass at an angle of incidence of $50^{\circ}$.

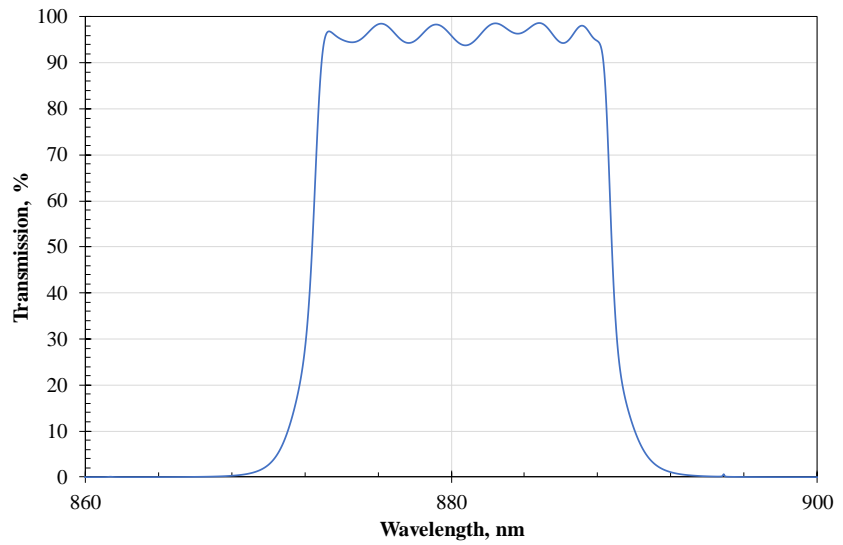

Fig. 2. Spectral dependence of the transmission of the designed bandpass for $\mathrm{a} 50^{\circ}$ angle of incidence.

One can see that high transmission in bandpass higher than $91.7 \%$ can be achieved, however the steepness of the edge of the bandpass between $10 \%$ and $80 \%$ is slightly higher than the specification and is equal to $2.3 \mathrm{~nm}$ but is still acceptable.

Such a filter was then fabricated using a Bühler HELIOS 800. " where low and high refractive index materials were both deposited through Plasma Assisted Reactive Magnetron Sputtering (PARMS). The main chamber is subdivided into different treatment zones, two dedicated for dielectric materials (MF magnetron sputtering), and one for oxygen plasma assistance (PBS). The substrates are set on a 12-position rotating sample holder (rotation at $240 \mathrm{rpm}$ ). According to the deposited material, either low or high index, the corresponding MF magnetron sputtering cathode is switched on. The oxygen plasma assistance is used for densification of the coating and also to respect the stoichiometry of the layers. Typical deposition rates of low and high index materials are respectively around $0.40-0.45 \mathrm{~nm} . \mathrm{s}^{-1}$ and $0.50-0.60 \mathrm{~nm} . \mathrm{s}^{-1}$. The control of the thickness of each of the layers was carried out using an OMS 5100 optical monitoring system. Efficient optical monitoring based on 5 different monitoring test glasses was developed and validated using internal virtual deposition process software. Based on the typical noise parameters of the OMS 5100, these simulations show that errors on the thickness of each layer should be kept below $1 \%$.

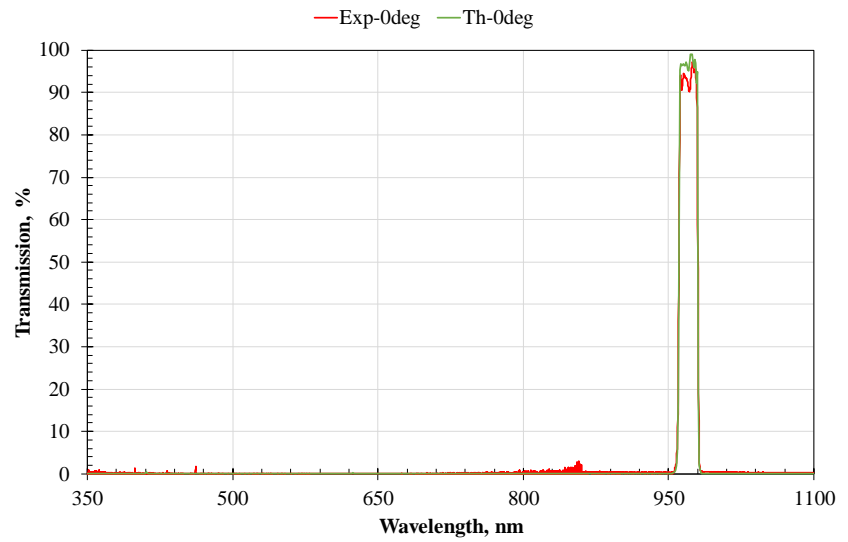


Fig. 3. Spectral performances of the fabricated bandpass filter measured using a Perkin Elmer Lambda 1050 spectrophotometer at $0^{\circ}$.

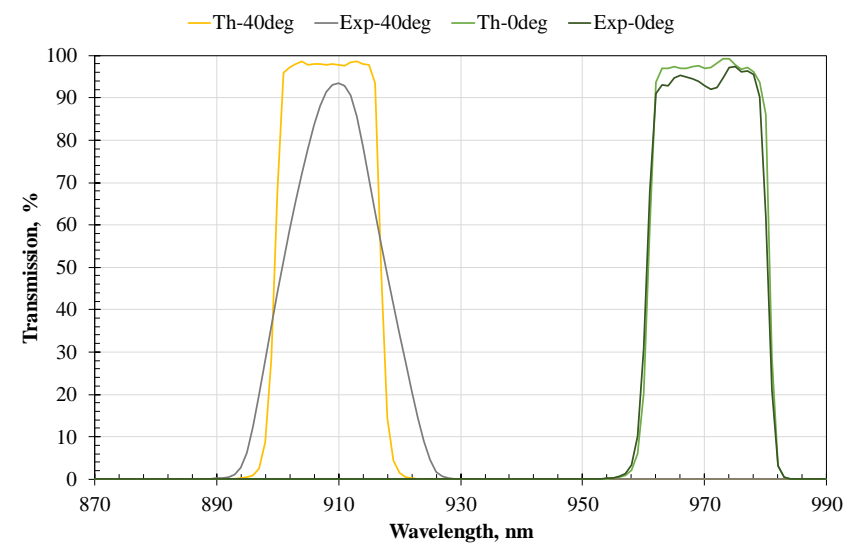

Fig. 4. Spectral performances of the fabricated bandpass filter measured using a Perkin Elmer Lambda 1050 spectrophotometer at $0^{\circ}$ and $40^{\circ}$.

The performances of such a filter were then evaluated using a Perkin Elmer Lambda 1050 spectrophotometer. Figures 3 and 4, shows the typical spectral profiles that have been measured over a broad spectral range at normal incidence and around the bandpass regions for two different angles of incidence of $0^{\circ}$ et $40^{\circ}$ for natural light polarization, i.e. $50 \% \mathrm{~S}$ and $50 \% \mathrm{P}$ polarizations. One can see that the performances of the filter could be accurately measured at $0^{\circ}$ with a spectrophotometer, confirming that the errors on the thickness of each layer of the filter were kept very low due to the large error sensitivity of the design. However, for a larger angle of incidence $\left(40^{\circ}\right)$, one can see large discrepancies between theoretical and experimental curves, even when changing the width of the slits (resolution was decreased down to $0.1 \mathrm{~nm}$ ). This discrepancy is not due to a limited spectral resolution of the spectrophotometer but due to the beam divergence of the measurement beam. Indeed, the beam of a spectrophotometer is multimode and not perfectly collimated. The typical divergence is between 2 and $3^{\circ}$. Thin-film filters are known to be mildly dependent on the divergence at normal incidence but this sensitivity severely increases with incidence. At an angle of incidence of $40^{\circ}$, the measured transmission is the average transmission over the acceptance cone of $40^{\circ} \pm$ divergence. Due to the quick and large shift of the bandpass central wavelength on angle of incidence for this range of incidence, the measured transmission is highly affected and does not allow matching the theoretical profile. To overcome this limitation, people generally calculate theoretically the new profile taking into account this divergence in order to confirm that the measured spectra are accurate. But this indirect method is generally quite challenging with a commercial system as the divergence is not constant and depends on the slit width, the beam diameter and various intrinsic parameters of the spectrophotometer associated with the monochromator performances. In this work, we developed a dedicated setup in order to perform these spectral measurements. The aim of this setup was to match the experimental conditions, as close as possible, to theoretical conditions of the calculation. Among those, we can cite:

- monochromatic measurement conditions,
- low beam divergence as close to a plane wave,

- small beam diameter in order to perform a local measurement and minimize the effect of the filter non uniformity,

- high signal to noise ratio.

It is interesting to note that meeting all the conditions will result in a compromise. For example, decreasing the spectral resolution generally results in lower signal to noise ratio. Similarly, decreasing the beam diameter will result in increasing the beam divergence. We represented in Figure 5, a scheme of the setup that we developed.

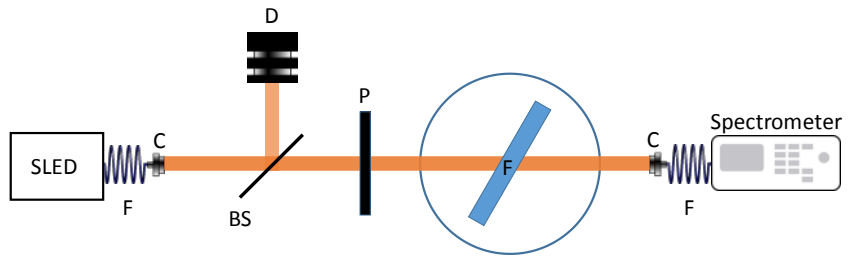

Figure 5. Setup for the measurement of the transmission of angularly bandpass filters. SLED is a light source, $\mathrm{F}$ are single mode fibers, $\mathrm{C}$ is a collimator, $\mathrm{BS}$ is a beamsplitter, $\mathrm{D}$ is a photodiode-type detector, $\mathrm{P}$ is a polarizer, OSA is a spectrometer and $\mathrm{F}$ the components to be characterized.

This setup has the following characteristics:

1. The light source (SLED) is a broadband source (superluminescent diode) coupled in a single mode fiber (F). It is thus possible to guarantee both a high spectral power density and a monomode transverse intensity distribution. It is centered at $920 \mathrm{~nm}$, the spectral width at $-3 \mathrm{~dB}$ is larger than $100 \mathrm{~nm}$, and the total power at the output of the single mode fiber is $\sim 5 \mathrm{~mW}$.

2. An emission collimator (C) is then used to generate a lowaberration Gaussian single-mode collimated beam (TEM ${ }_{00}$ ) on the spectral domain of interest $\left(\mathrm{M}^{2}<1.2\right)$. The beam diameter was fixed to $2 \mathrm{~mm}$ in order to secure low beam divergence $(<$ $1 \mathrm{mrad}$ ) and small probed area.

3. A linear polarizer $(\mathrm{P})$ mounted on a rotation plate controls the polarization at the output of the input collimator.

4. A reference channel (BS $+D)$ was used to measure the possible low intensity fluctuations of the source.

5. A sample holder system including a rotation plate makes it possible to control the angle of incidence of the beam on the component to be characterized and translational plates make it possible to scan the measurement zone on the sample if necessary.

6. A collimator of reception (C) is then used to collect all the light transmitted by the system. This collimator is fixed on a translational stage in order to compensate for the deviation of the beam transmitted by the component due to the effects of refraction in the substrate in order to maximize the collected flux. This displacement can be either adjusted by maximization of the measured power or predicted based on the substrate material and thickness.

7. A single mode fiber is then used to collect the light transmitted by the component and is sent to an Ando optical spectrum analyzer (OSA). The use of a single mode fiber in combination with the OSA allows to optimize the spectral resolution of the 
measurement down to $0.15 \mathrm{~nm}$ over the measurement spectral range.

A measurement sequence then consists in carrying out, for each of the polarizations, a spectral measurement of the intensity transmitted in the air $\mathrm{I}_{100 \%}$, then a similar measurement in the case where the beam is blocked $\left(\mathrm{I}_{0 \%}\right)$, and finally a measurement in transmission for each angle of incidence and with an optimized position of the collecting collimator (I). Transmission is then obtained by:

$$
\mathrm{T}=\frac{\mathrm{I}-I_{0 \%}}{I_{100 \%}-I_{0 \%}}
$$

This equation does not take into account the reference arm of the setup. Indeed, each of the intensities defined above is in fact the ratio between the intensities measured by the measurement channel (OSA) and those measured by the reference channel (D).

Preliminary measurements were first carried out on the filter without controlling the polarization. In these conditions, the light is partially polarized with an approximate ratio: 80\%-S / 20\%-P. Figures6 provides an illustration of the spectral performances of the filter in linear and log scales and a comparison of the theoretical performances when illuminated using this polarization ratio.
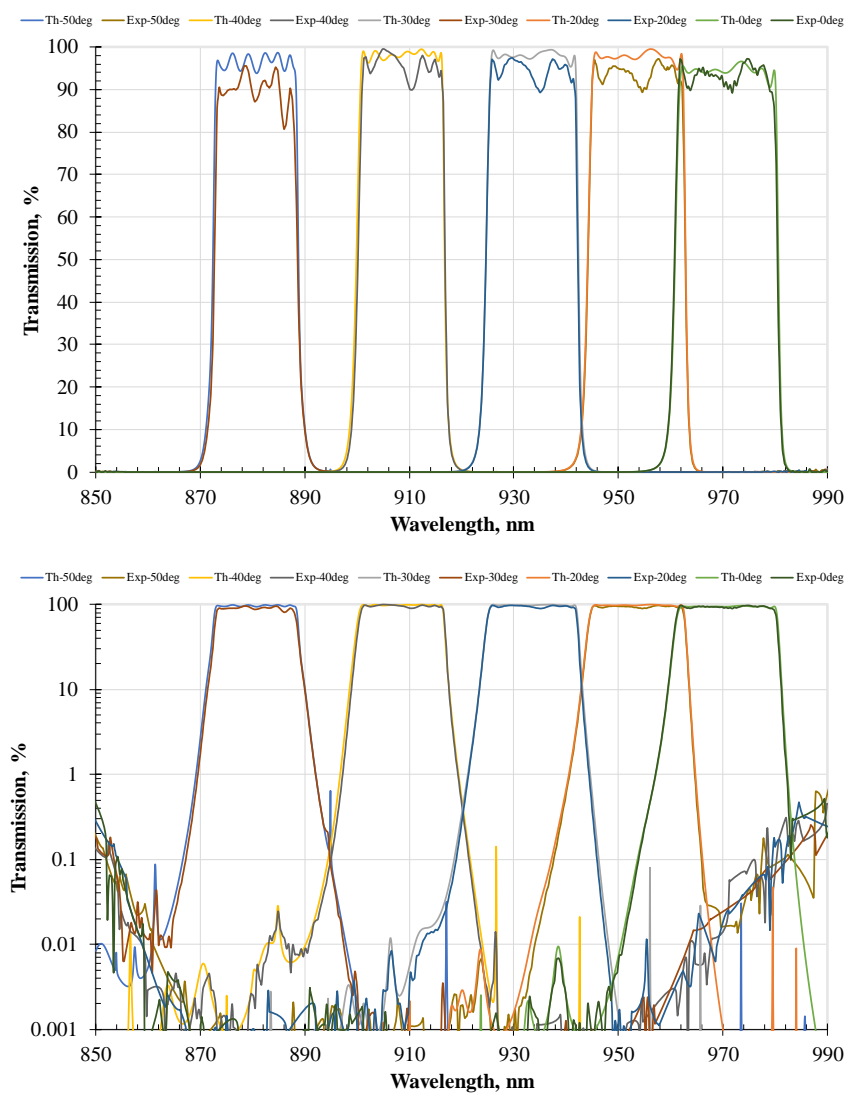

Figure 6. Spectral dependence of the transmission measured on the bandpass filter for different angles of incidence between 0 and $50^{\circ}$ and comparison with theoretical transmission curves - Top: Linear scale, bottom: Log scale.

One can see that without any recalculation or reverse engineering, there is a close-to-perfect match between the theoretical and experimental transmission measurements. One can also see that there is a good agreement both in linear and log scales, i.e. in high and low transmittance regions. Experimental curves show larger oscillations in the bandpass region most probably associated with manufacturing errors. These oscillations are real features of the filter, i.e. repeatable and not associated with noise fluctuations. Finally, absolute transmission precision is estimated to be within $5 \%$ and could be improved by further improvement of the measurement system.

In order to better understand the effect of polarization, we also measured the transmission of the filter at $50^{\circ}$ angle of incidence for both $\mathrm{S}$ and $\mathrm{P}$ polarizations by inserting a polarizer before the sample to be measured. Figure 7 provides an illustration of the spectral performances of the filters at $50^{\circ}$ in $\mathrm{S}$ and $\mathrm{P}$ polarizations in log scale. One can see that broadening effect in P-polarization in comparison with S-polarization is clearly illustrated experimentally and good agreement between theory and experiment is also observed. One can even see the small parasitic peak at $895 \mathrm{~nm}$ in both experimental and theoretical curve in log scale confirming that both experimental fabrication and characterization have been performed with very minimal errors.

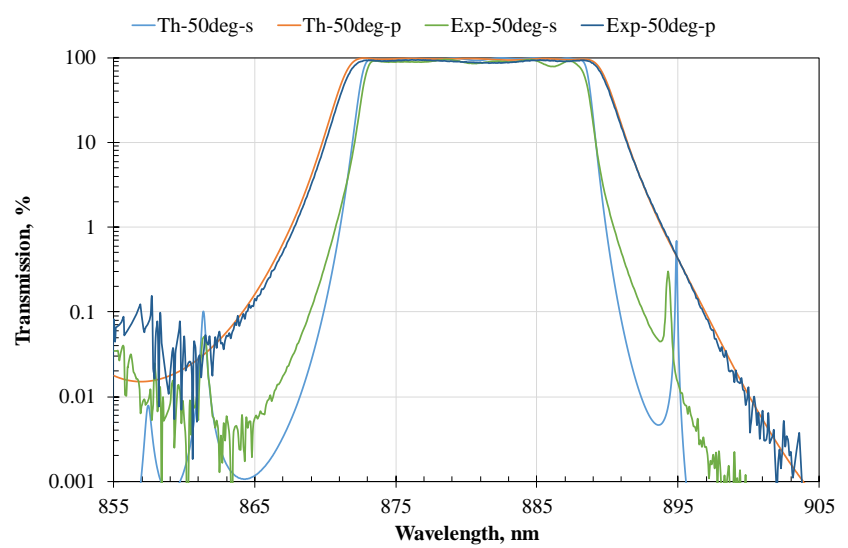

Figure 7. Spectral dependence of the transmission measured on the bandpass filter at $50^{\circ}$ angle of incidence in $s$ and p polarizations and comparison with theoretical transmission curves - Log scale.

We have shown the design, the manufacturing and the characterization of an angularly tunable bandpass filter that shows minimal distortions of the bandpass with angle tuning between 0 and $50^{\circ}$ and a perfect agreement between theoretical and experimental results.

\section{References}

1. https://www.opticsbalzers.com/en/coating/dielectric-coatings/

2. https://www.edmundoptics.com/c/optical-filters/610/\#

3. Philip Baumeister, Appl. Opt. 37, 6609-6614 (1998)

4. Paras N. Prasad, Introduction to Biophotonics, John Wiley \& Sons, Inc. P. 235 (2003) - DOI:10.1002/0471465380

5. Le Goff, R., Badoil, B., Fuss, P. Tanguy, F., and Etcheto, P., Proc. SPIE 8176,

Sensors, Systems, and Next-Generation Satellites XV, 817618 (2011)

6. Haruo Takashashi, Appl. Opt. 34, 667-675 (1995)

7. Mi-Yun Jeong and Jin Yeob MangAppl. Opt. 57, 1962-1966 (2018)

8. I. C. Chang, I. C. Chang, Optical Engineering 20(6), 206824 (1981). 
9. R. Parmentier, F. Lemarchand, M. Cathelinaud, M. Lequime, C. Amra, S. Labat, S. Bozzo, F. Bocquet, A. Charaï, O. Thomas and C. Dominici, Appl. Opt., 41, 3270-3276 (2002)

10. R. Parmentier and M. Lequime, Opt. Letters, 28, 728-730 (2003) and Opt. Letters, 28, 1279 (2003)

11. H. A. Macleod, Thin-Film Optical Filters, 4th ed. (CRC Press, 2010), P. 329 and 31.

12. https://www.semrock.com/filters.aspx

13. https://www.optilayer.com/ 


\section{Full References:}

1. https://www.opticsbalzers.com/en/coating/dielectric-coatings/

2. https://www.edmundoptics.com/c/optical-filters/610/\#

3. Philip Baumeister, "Bandpass filters for wavelength division multiplexingmodification of the spectral bandwidth," Appl. Opt. 37, 6609-6614 (1998)

4. Paras N. Prasad, Introduction to Biophotonics, John Wiley \& Sons, Inc. P. 235 (2003) - DOI:10.1002/0471465380

5. Le Goff, R., Badoil, B., Fuss, P. Tanguy, F., and Etcheto, P., "Recent developments of multispectral filter assemblies for CCD, CMOS and bolometer," Proc. SPIE 8176, Sensors, Systems, and Next-Generation Satellites XV, 817618 (October 03, 2011)

6. Haruo Takashashi, "Temperature stability of thin-film narrow-bandpass filters produced by ion-assisted deposition," Appl. Opt. 34, 667-675 (1995)

7. Mi-Yun Jeong and Jin Yeob Mang, "Continuously tunable optical notch filter and band-pass filter systems that cover the visible to near-infrared spectral ranges," Appl. Opt. 57, 1962-1966 (2018)

8. I. C. Chang, I. C. Chang, "Acousto-Optic Tunable Filters," Optical Engineering 20(6), 206824 (1 December 1981).

9. R. Parmentier, F. Lemarchand, M. Cathelinaud, M. Lequime, C. Amra, S. Labat, S. Bozzo, F. Bocquet, A. Charaï, O. Thomas and C. Dominici, "Piezoelectric Tantalum Pentoxide Studied for Optical Tunable Applications," Appl. Opt., 41, 3270-3276 (2002)

10. R. Parmentier and M. Lequime, "Substrate-Strain-Induced Tunability of Dense Wavelength-Division Multiplexing Thin-Film Filters," Opt. Letters, 28, 728-730 (2003) and Opt. Letters, 28, 1279 (2003)

11. H. A. Macleod, Thin-Film Optical Filters, 4th ed. (CRC Press, 2010), P. 329 and 31.

12. https://www.semrock.com/filters.aspx

13. https://www.optilayer.com/ 